\title{
Assessing Confidence in Parallel Simulation Results
}

\author{
Fabian M. Uriarte \\ Center for Electromechanics \\ The University of Texas at Austin \\ Austin, TX, USA \\ f.uriarte@,cem.utexas.edu
}

\author{
Robert E. Hebner \\ Center for Electromechanics \\ The University of Texas at Austin \\ Austin, TX, USA \\ r.hebner@cem.utexas.edu
}

\begin{abstract}
To accelerate the simulation of microgrid behavior, a parallel power system solver is being developed. The solver (CEMSolver) aims to accelerate the simulation of large-scale shipboard power system models created in MATLAB/Simulink with the SimPowerSystems blockset. While speedups of 50x have been reported, there remains insufficient confidence in the simulation results. This paper presents a point-by-point comparison of the results produced by $M A T L A B /$ Simulink 2012a and in parallel by CEMSolver. A large shipboard power system model is used for the comparison. It is concluded that, while the results show differences related to integration methods, all reported measurements of instantaneous voltage and current waveforms are within reasonable agreement.
\end{abstract}

\section{INTRODUCTION}

Simulation experience in the Electric Ship Research and Development Consortium (ESRDC [1]) bears testimony that simulation run times of large-scale shipboard power system models are too time consuming to be useful in early-stage design trades. To reduce this run time, one could either use a faster processor approach, such as field-programmable gate array or modify the software to be efficient in multicore systems. Given the continued growth of multicore systems, it was decided to develop a Windows-based multicore solution.

Speed is important, but it cannot be achieved at the expense of accuracy. Accuracy is assessed herein by taking the absolute value of the point-by-point arithmetic difference between the results provided by MATLAB/Simulink and CEMSolver. The results provided by MATLAB/Simulink are believed to be single-matrix, non-parallel results, whereas the results produced by CEMSolver are produced in parallel by tearing the power system model into four partitions. This provides a sense of the relative accuracy with respect to a commonly used code.

The computer used to simulate the shipboard power system presented in this work was a Windows 7, Dell Precision T7500 workstation with 12 GB of RAM and an Intel Xeon E5630, $2.53 \mathrm{GHz}$, quad-core processor. The partitioning approach used to parallelize power system simulations was reported in $[2,3]$, and is omitted in this work. (Further information on the partitioning strategy and implementation is available in [4].)

\section{SHIPBOARD POWER SYSTEM}

Confidence in parallel (partitioned) simulation results is assessed by parallelizing the simulation of a notional Navy shipboard power system model and comparing the results (point-by-point) against the results produced by MATLAB/Simulink 2012a. The model presents characteristics of Navy shipboard power systems (AC-radial type), but does not represent any particular system. The information in [5-9] was used to construct said notional model in MATLAB/Simulink [10] using the SimPowerSystems blockset as the library of most power apparatus models.

The one-line diagram of the shipboard power system model is shown in Figure 1. This model represents the electric distribution (ship service) side of an electromechanical shipboard, and it is not related to an integrated power system or all-electric ship [11]. The power apparatus shown in Figure 1 were modeled as follows. The generators were modeled as three-phase voltage sources behind their sub-transient impedances. Cables were modeled as nominal $\pi$-sections including line-to-line capacitance. (No stray capacitance to the ship hull was considered, even though such capacitance is present [12].) The cable impedances were taken from Table XIII in $[6,7]$ ( $L S T S G U$ three-conductor, shipboard power cable, $450 \mathrm{~V}$, three-phase, $60 \mathrm{~Hz}$ ). The transformers were modeled as three single-phase units rated at $25 \mathrm{kVA}, 450: 120$ $\mathrm{V}$ each. The transformer banks were connected in delta on both sides [9].

The loads were modeled as static impedance loads, and are tabulated in TABLE I by type. The columns in TABLE I indicate the volt-ampere rating in $\mathrm{kVA}$, the power consumption in $\mathrm{kW}$, the reactive power draw or injection in $\mathrm{kVar}$, the power factor (PF), and the full-load ampere (FLA) draw. The location of these loads is shown in Figure 1. Loads of type L were modeled as static, series RL impedances. Loads of type $\mathrm{M}$ were modeled as three-phase, six-pulse, selfcommutated rectifiers serving a dc resistive load. These 19 rectifiers are comprised of $19 \times 6=114$ diodes, which were purposefully considered to increase simulation complexity and make the accuracy assessment more realistic. 
TABLE I LOAD RATINGS AND TOTAL SYSTEM LOAD

\begin{tabular}{|c|c|c|c|c|c|}
\hline Name & kVA & $\mathrm{kW}^{*}$ & kVar & $\mathrm{PF}^{\wedge}$ & FLA \\
\hline L01 & 6.60 & 6.60 & - & 1.00 & 8.48 \\
\hline L02 & 6.60 & 6.60 & - & 1.00 & 8.48 \\
\hline L03 & 6.60 & 6.60 & - & 1.00 & 8.48 \\
\hline L04 & 11.77 & 10.00 & 6.20 & 0.85 & 15.11 \\
\hline L05 & 35.29 & 30.00 & 18.59 & 0.85 & 45.33 \\
\hline L06 & 35.29 & 30.00 & 18.59 & 0.85 & 45.33 \\
\hline L07 & 38.11 & 36.20 & 11.90 & 0.95 & 48.95 \\
\hline L08 & 38.11 & 36.20 & 11.90 & 0.95 & 48.95 \\
\hline L09 & 38.11 & 36.20 & 11.90 & 0.95 & 48.95 \\
\hline L10 & 70.00 & 42.00 & 56.00 & 0.60 & 89.92 \\
\hline L11 & 190.53 & 181.00 & 59.49 & 0.95 & 244.73 \\
\hline L12 & 315.79 & 300.00 & 98.61 & 0.95 & 405.64 \\
\hline L13 & 333.33 & 300.00 & 145.30 & 0.90 & 428.18 \\
\hline M01 & 4.55 & 4.55 & - & 1.00 & 5.84 \\
\hline M02 & 4.55 & 4.55 & - & 1.00 & 5.84 \\
\hline M03 & 42.87 & 42.87 & - & 1.00 & 55.07 \\
\hline M04 & 52.05 & 52.05 & - & 1.00 & 66.85 \\
\hline M05 & 52.05 & 52.05 & - & 1.00 & 66.85 \\
\hline M06 & 52.05 & 52.05 & - & 1.00 & 66.85 \\
\hline M07 & 52.05 & 52.05 & - & 0.86 & 66.85 \\
\hline M08 & 86.74 & 86.74 & - & 1.00 & 111.42 \\
\hline M09 & 86.74 & 86.74 & - & 1.00 & 111.42 \\
\hline M10 & 134.82 & 134.82 & - & 1.00 & 173.18 \\
\hline M11 & 134.82 & 134.82 & - & 1.00 & 173.18 \\
\hline M12 & 134.82 & 134.82 & - & 1.00 & 173.18 \\
\hline M13 & 134.82 & 134.82 & - & 1.00 & 173.18 \\
\hline M14 & 134.82 & 134.82 & - & 1.00 & 173.18 \\
\hline M15 & 134.82 & 134.82 & - & 1.00 & 173.18 \\
\hline M16 & 207.22 & 207.22 & - & 1.00 & 266.18 \\
\hline M17 & 207.22 & 207.22 & - & 1.00 & 266.18 \\
\hline M18 & 207.22 & 207.22 & - & 1.00 & 266.18 \\
\hline M19 & 207.22 & 207.22 & - & 1.00 & 266.18 \\
\hline T01 & 75.00 & 70.00 & 26.93 & 0.93 & 89.92 \\
\hline T02 & 75.00 & 70.00 & 26.93 & 0.93 & 89.92 \\
\hline T03 & 75.00 & 70.00 & 26.93 & 0.93 & 89.92 \\
\hline T04 & 75.00 & 70.00 & 26.93 & 0.93 & 89.92 \\
\hline T05 & 75.00 & 70.00 & 26.93 & 0.93 & 89.92 \\
\hline T06 & 75.00 & 70.00 & 26.93 & 0.93 & 89.92 \\
\hline T07 & 75.00 & 70.00 & 26.93 & 0.93 & 89.92 \\
\hline T08 & 75.00 & 70.00 & 26.93 & 0.93 & 89.92 \\
\hline T09 & 75.00 & 70.00 & 26.93 & 0.93 & 89.92 \\
\hline T10 & 75.00 & 70.00 & 26.93 & 0.93 & 89.92 \\
\hline $\mathrm{T} 11$ & 75.00 & 70.00 & 26.93 & 0.93 & 89.92 \\
\hline Total: & 4,023 & 3,863 & 438 & & 4,107 \\
\hline
\end{tabular}

\section{SimUlation RESUltS}

Figure 2-Figure 7 show the voltage and current measurements at the three locations indicated by Figure 1 (measurements 1, 2, and 3). In all figures, the left-most column shows the voltage (a) and current (c) produced by Simulink (fixed-step discrete solver). The center column shows the voltage (b) and current (e) produced by CEMSolver, which uses root matching [13-15] integration, and parallelizes the simulation results using four partitions. (The speedup for the power system model in Figure 1 was 20x, but it is not the focus of this work.) The right-most columns in Figure 2Figure 7 show the absolute value of the instantaneous (pointby-point) difference in voltage and current labeled as subfigures (c) and current (f).

The thick bidirectional arrows connecting sub-charts (a) and (b) or (d) and (e) (in all figures) suggest the visual impact of the result. For example, comparing Figure 2(a) and (b), there is a clear distinction between the results. Similarly, a comparison between Figure 3(d) and (e) leads to a perceived agreement in results. Sincevisual perceptions can be deceptive, sub-charts (c) and (f) in all figures, report the actual difference in results. The absolute value of the instantaneous difference is calculated as $\left|x_{i}-x_{j}\right|$, where $x_{i}$ represents a data point (voltage or current) produced by Simulink and $x_{j}$ the corresponding data point produced by CEMSolver. Although there are other approaches to show differences, such as averages, rms, or percent errors, the instantaneous difference approach was used for two reasons. First, it does not filter spikes, as do estimates based on moving windows. Second, the word error is avoided as it is not certain which result set is correct (Simulink's or CEMSolvers'). It is likely that neither result set is correct, as both can only approximate actual physical behavior.

The simulation time step used for the comparisons was $\Delta t=1 \mu \mathrm{s}$ and the stop time was $t_{\text {stop }}=25 \mathrm{~ms}$. This time step value is somewhat short for electric power networks. However, anticipating higher-frequency silicon-carbide converters will require shorter time step than the usual $\Delta t=50$ $\mu \mathrm{s}$, so the choice of $\Delta t=1 \mu \mathrm{s}$ seemed appropriate. Additionally, $\Delta t=1 \mu \mathrm{s}$ increases the accuracy of the results produced by Simulink's Tustin and backward Euelr integration methods. It is also understood that larger values of $\Delta t$ can lead to diverging differences in results $[16,17]$.

The following subsections discuss the results obtained at measurement locations 1, 2, and 3 shown in Figure 1. The result sets compare the results produced by Simulink using the Tustin and backward Euler methods against the parallel results by CEMSolver using root matching integration.

\section{A. Simulation1 (Fig. 2 and Fig. 3)}

Figure 2(a) shows an initial distortion at the terminal voltage of the generator labeled GEN1. These oscillations are deemed numerical chatter, and appear often when using the Tustin (or trapezoidal rule) integration method in power networks with switches. Methods to mitigate these spurious oscillations are well understood [17-20], but based on this result, they may not be part of the current release of Simulink (2012a). Figure 2(b) shows the same calculated results produced in parallel with CEMSolver. These results do not exhibit chatter. The absolute value of the instantaneous difference between Figure 2(a) and Figure 2(b) is shown in Figure 2(c). As seen, the difference in voltage reaches several hundred volts, but it dampens as the simulation advances.

Figure 2(d) shows the currents measured at GEN1. The currents show physical harmonic distortion consistent with a system heavily loaded with three-phase six-pulse rectifiers (loads M01 through M19 in TABLE I). There is an initial (bare) resonance in the first positive cycle of $i_{c}$ (blue waveform, Figure 2(d)) also seen in the results produced by CEMSolver (Figure 2(e)). The difference in resonance amplitude is shown in Figure 2(d), which reaches several hundred amps for the first $10 \mathrm{~ms}$ of simulation.

The simulation results in Figure 2 were produced using Tustin integration. The same calculated results shown when using backward Euler integration in Simulink are shown in Figure 3. Use of this third integration technique increases confidence in the parallel results produced by CEMSolver. 
For example, comparing Figure 2(a) (Simulink, Tustin) and Figure 3(a) (Simulink, backward Euler), the voltage chatter is no longer present. The results produced by the backward Euler technique agree more with the parallel results produced with CEMSolver. The absolute value of the difference of voltages when using Simulink's backward Euler method and CEMSolver's root matching method is shown in Figure 3 (c). The difference is significantly reduced when compared to Figure 2(c). (The scale in Figure 3 (c) and (f) was adjusted with respect to Figure 2 (c) and (f) for convenience). It is interesting to realize that use of another (third) integration method serves as a discriminator in elevating confidence in the parallel results produced by CEMSolver.

A comparison of the currents measured at GEN1 when using Simulink's backward Euler method and CEMSolver's root matching method is shown Figure 3(f). The difference in results is less pronounced than in Figure 2(f). While the differences are reduced, they still exist. The differences are likely due to the integration method as the root-matching method represents more accurately the poles and zeros in the discrete frequency domain [14] than does the truncatedTaylor-series integration methods such as Tustin (or trapezoidal rule) and backward Euler. Sincethe absolute value of the instantaneous difference is small, it is said that there is a perceived agreement between the results in Figure 3 (indicated by thick bi-directional arrows).

\section{B. Simulation 2 (Fig. 4 and Fig. 5)}

Figure 4 shows the three-phase voltages and currents measured at the terminals of load M11 (zone E3 in Figure 1). The voltage waveforms in Figure 4(a) and Figure 4(b) show a distinction that is clearly visible. The distinction is produced by the intermittent oscillations produced by Simulink's Tustin integration method and does not dampen. Referring to the differences in voltage in Figure 4(c), although these differences appear small, they reach $\sim 40 \mathrm{~V}$ in all three phases. Moreover, the differences occur in steady state, which suggests that these differences will likely diverge under abrupt system changes (e.g., a system fault).

Figure 4(d) and (e) show the three-phase current entering M11, which are currents entering one of the nineteen sixpulse, self-commutated, three-phase rectifiers serving dc loads at $450 \times 1.35 \approx 600 V_{d c}$. (Load information was given in TABLE I.) There is a perceived agreement in the currents even though the voltages show clear distinctions. The instantaneous difference between the current is shown in Figure 4(f), which disagrees by a few amps during diode commutations. The exact source of the difference is likely related to both the integration method (Tustin vs. root matching) and to how diodes are treated in Simulink vs. how they are treated in CEMSolver. In Simulink diodes are treated current sources. In CEMSolver, they are treated as piecewiselinear resistors. To reduce the impact of discretization method, the same result set was reproduced using the backward Euler integration method in Simulink and is shown in Figure 5.

Figure 5 shows the result sets of Figure 4 using the backward Euler method. Comparing Figure 4(c) against Figure 5(c) shows that the change in integration method (from
Tustin to backward Euler) significantly reduced voltage difference. Similarly, a comparison of Figure 4(f) and Figure 5(f) also shows the same reduction effect for the current. Figure 5(c) and Figure 5(f) show that the differences between the parallel results of CEMSolver and Simulink are small, but are not negligible. The differences in Figure 4 were attributed to numerical chatter and switch model. The differences in Figure 5 appear to be related only to how the diodes are modeled.

Switch modeling is outside the scope of this work, but its impact includes piecewise linearization and how time interpolation is implemented in code. It is not clear how Simulink addresses time interpolation, but in the general sense, time interpolation [21-23] is a rollback routine used by time domain solvers to address states transitions between time step intervals. The differences related to switching in Figure 5 are likely to be caused by these two modeling aspects related to switching. Despite these modeling differences, the voltage and current waveforms shown in Figure 5 are in reasonable agreement.

\section{Simulation 3 (Fig. 6 and Fig. 7)}

Figure 6 shows the voltages measured on the secondary side of the transformer labeled T07 (450:120 V, $\Delta-\Delta, 3 \times 25-$ $\mathrm{kVA}$ single-phase units totaling a $75 \mathrm{kVA}$ rating) when using the Tustin method. The voltages $(120 \mathrm{~V}$ side) shown in Figure 6(a) and Figure 6 (b) are in reasonable agreement, having differences of less than $1 \mathrm{~V}$ (Figure 6(c)).

It is also interesting to note that the absolute value of the instantaneous differences vary by measurement location. That is, Figure 2(c) and Figure 4(c) show differences much higher than Figure 6(c) do. Referring to Figure 1, there are hundreds of locations to place measurements; however, only three were selected. Ideally, comparisons would be made at every junction interconnecting two or more components (i.e., all switchboards, load centers, and places where two or more pieces of power apparatus interconnect). However, this would require adding too many three-phase voltage and current measurements in Simulink and would significantly increase simulation runtime - especially at $\Delta t=1 \mu \mathrm{s}$. Thus, a ubiquitous comparison of results is an interesting academic exercise, but is impractical to exercise on a regular basis.

The transformer currents shown in Figure 6(d) and Figure 6(e) are in reasonable agreement, but differences exist as shown in Figure 6(f). The differences relate to the transformer model itself and its load. The three-phase transformer models include winding series (RL) impedances and core magnetizing losses (parallel RL branch). The transformer load is a parallel $\mathrm{RC}$ branch drawing $\leq 75 \mathrm{kVA}$ with a leading power factor (see TABLE I). As deduced so far, everywhere there are RL or RC branch pairs, differences exist due to the difference in integration methods used by Simulink and CEMSolver. Despite these differences in integration methods, the results exhibit good agreement.

Finally, a comparison of the secondary-side transformer voltage and current is shown in Figure 7. Both voltage and current are in reasonable agreement. The absolute value of the difference in Figure 7(c) and (f) is about 2/3 of what they were 
in Figure 6. This reduction in the point-by-point difference confirms the importance in comparing the parallel results against the two integration methods available in Simulink. If the differences reduce by changing integration method, it is clear the differences are due, at least in part, to the differences in integration methods rather than differences in modeling. In both Figure 6 and in Figure 7, the results appear to be in reasonable agreement.

\section{CONCLUSIONS}

Two tiers are defined for the development of a parallel solver: speed and accuracy. Speed is at the forefront of research and the one most likely to receive attention first. Accuracy is an assumed byproduct of the former. However, confidence in the accuracy is fundamental to developing a solver and is equal in importance to speed. This work showed progress toward elevating confidence in the results produced in parallel by CEMSolver.

The Tustin integration method of Simulink makes the parallel results produced by CEMSolver appear to be in some disagreement. However, use of the backward Euler method in Simulink reduces the difference and so increases confidence in that the parallel results produced by CEMSolver are consistent with what can be achieved using Simulink.

The root matching method produces results with accuracy closer to the Tustin method in sinusoidal networks, but closer to the backward Euler method in switching networks. It is not clear which integration method is correct (Tustin, backward Euler, and root matching), but the consistent trends in the results provide confidence in the parallel results produced by CEMSolver.

Moreover, the reported level of agreement has been reached by this work despite key differences, including:

- Different development teams (private industry vs. academia),

- Different formulation methods (state-space vs. nodal),

- Different solution approach (unpartitioned vs. parallel),

- Different integration method (Tustin or backward Euler vs. root matching),

- Switch-model differences (current sources vs. piecewise linear), and

- Other differences not readily apparent (e.g., internal programming optimizations or inclusion of parasitic impedances to prevent numeric instability).

An important observation is that confidence is limited by the limited number of points of comparison within the model. The model considered herein is too large to use ubiquitous three-phase instantaneous voltage and current comparisons. The comparison locations in Figure 1 were chosen to highlight different circuit behaviors. Comparison point 1 was selected because differences at this location likely follow an aggregate trend. Comparison point 2 was selected to highlight the impact of switching transients. Comparison point 3 was selected behind a step down transformer to explore whether measurements were affected by magnetizing branches.

The three locations were arbitrarily selected and resulted in sufficient agreement in all cases. It is likely that other random comparison locations will lead to the same conclusion; however, a more deterministic approach would be to write a computer program to compare all data points at all buses in the power system model. Although highly desirable, this ideality is ambitious as run times would increase exponentially and would likely result in depleting local computer resources. That is, saving too much data at $\Delta t=1 \mu$ s can require storing billions of data points, and it is not always plausible.

\section{REFERENCES}

[1] R. E. Hebner, S. Dale, R. Dougal, S. Sudhoff, et al., "The U.S. ESRDC advances power system research for shipboard systems," in 43rd International Universities Power Engineering Conference, Padova, Italy, Sep. 1-4, 2008.

[2] F. M. Uriarte and R. Hebner, "Development of a multicore power system simulator for ship systems," in Electric Ship Technologies Symposium, Alexandria, VA, April 10-13, 2011, pp. 106-110.

[3] F. M. Uriarte, R. E. Hebner, and A. L. Gattozzi, "Accelerating the simulation of shipboard power systems," in Grand Challenges in Modeling \& Simulation, The Hague, Netherlands, June 27 - 30, 2011.

[4] F. M. Uriarte, Multicore Simulation of Power System Transients, $1^{\text {st }}$ ed. London: IET, 2013.

[5] IEEE Std 45, Recommended Practice for Electrical Installations on Shipboard.

[6] DoD Military Specification MIL-C-24643A (1994), Cables and Cords, Electric, Low Smoke, For Shipboard Use, General Specification for.

[7] DoD Military Handbook MIL-HDBK-299 (SH) (1989), Cable Comparison Handbook - Data Pertaining to Electric Shipboard Cable.

[8] IEEE Std 1709-2010, Recommended Practice for $1 \mathrm{kV}$ to $35 \mathrm{kV}$ Medium-Voltage DC Power Systems on Ships.

[9] "Naval Ships' Technical Manual (Ch. 320) - Electrical Power Distribution Systems (rev. 2)," ed, 1998 [Online]. Available: http://www.hnsa.org/doc/nstm/ch320.pdf.

[10] The MathWorks, Inc. (2010). Simulink 7 User's Guide. [Online]. Available: http://www.mathworks.com/help/toolbox/simulink/

[11] N. H. Doerry, "Next generation integrated power systems for the future fleet," in Corbin A. McNeill Symposium, Annapolis, MD, 2009.

[12] Naval Sea Systems Command NAVSEA SE000-00-EI M-100 (1983), Electronics Installation and Maintenance Book: Article 3-3.2 Misconceptions of a shipboard ungrounded system.

[13] N. R. Watson and G. D. Irwin, "Electromagnetic transient simulation of power systems using root-matching techniques," IEE Proceedings: Generation, Transmission and Distribution, vol. 145, pp. 481-486, 1998.

[14] N. Watson and J. Arrillaga, Power Systems Electromagnetic Transients Simulation, $1^{\text {st }}$ ed. London: IEE, 2003.

[15] J. M. Smith, Mathematical Modeling and Digital Simulation for Engineers and Scientists, 2nd ed. Washington, DC: John Wiley, 1987.

[16] W. Gao, E. Solodovnik, R. Dougal, G. Cokkinides, et al., "Elimination of numerical oscillations in power system dynamic simulation," in 18th IEEE Applied Power Electronics Conference and Exposition, Miami, FL, February, 2003, 2003, pp. 790-794.

[17] J. R. Marti and J. Lin, "Suppression of numerical oscillations in the EMTP," IEEE Trans. Power Systems, vol. 4, pp. 739-747, 1989.

[18] T. Funaki, T. Takazaqa, T. Tada, A. Kurita, et al., "A Study on the Usage of CDA in EMTP Simulations," in International Conference on Power Systems Transients, New Orleans, 2003, pp. 1-6.

[19] R. M. Kielkowski, Inside SPICE, $2^{\text {nd }}$ ed. New York: McGraw-Hill, 1998.

[20] J. A. Lima, "Numerical Instability due to EMTP-TACS Interrelation," EMTP Newsletter, vol. 5, pp. 21-33, 1985.

[21] P. Kuffel, K. Kent, and G. Irwin, "The Implementation and Effectiveness of Linear Interpolation Within Digital Simulation," in 
International Conference on Power System Transients, Lisbon, 1995, pp. 499-504.

[22] P. Kuffel, K. Kent, and G. Irwin, "The Implementation and Effectiveness of Linear Interpolation Within Digital Simulation," Electrical Power \& Energy Systems, vol. 19, pp. 221-227, 1997.
[23] L. R. Linares and J. R. Martí, "A resynchronization algorithm for topological changes in real time fast transients simulation," in Power Systems Computation Conference (PSCC'02), Sevilla, Spain, June 24$28,2002,2002$.

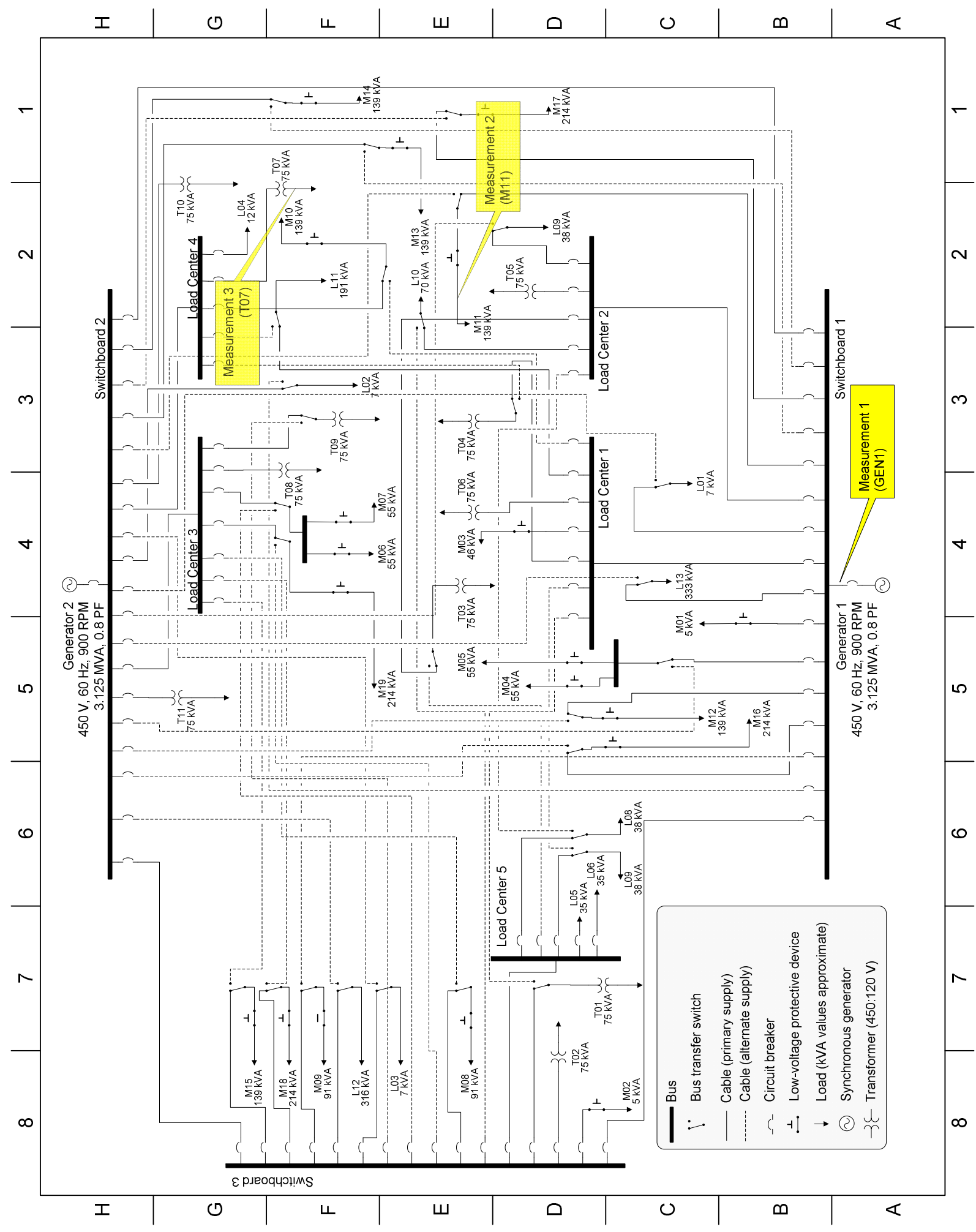

Figure 1 One-line diagram of notional shipboard power system (AC-radial, $450 \mathrm{~V}, 60 \mathrm{~Hz}$, three-phase, delta-ungrounded) 

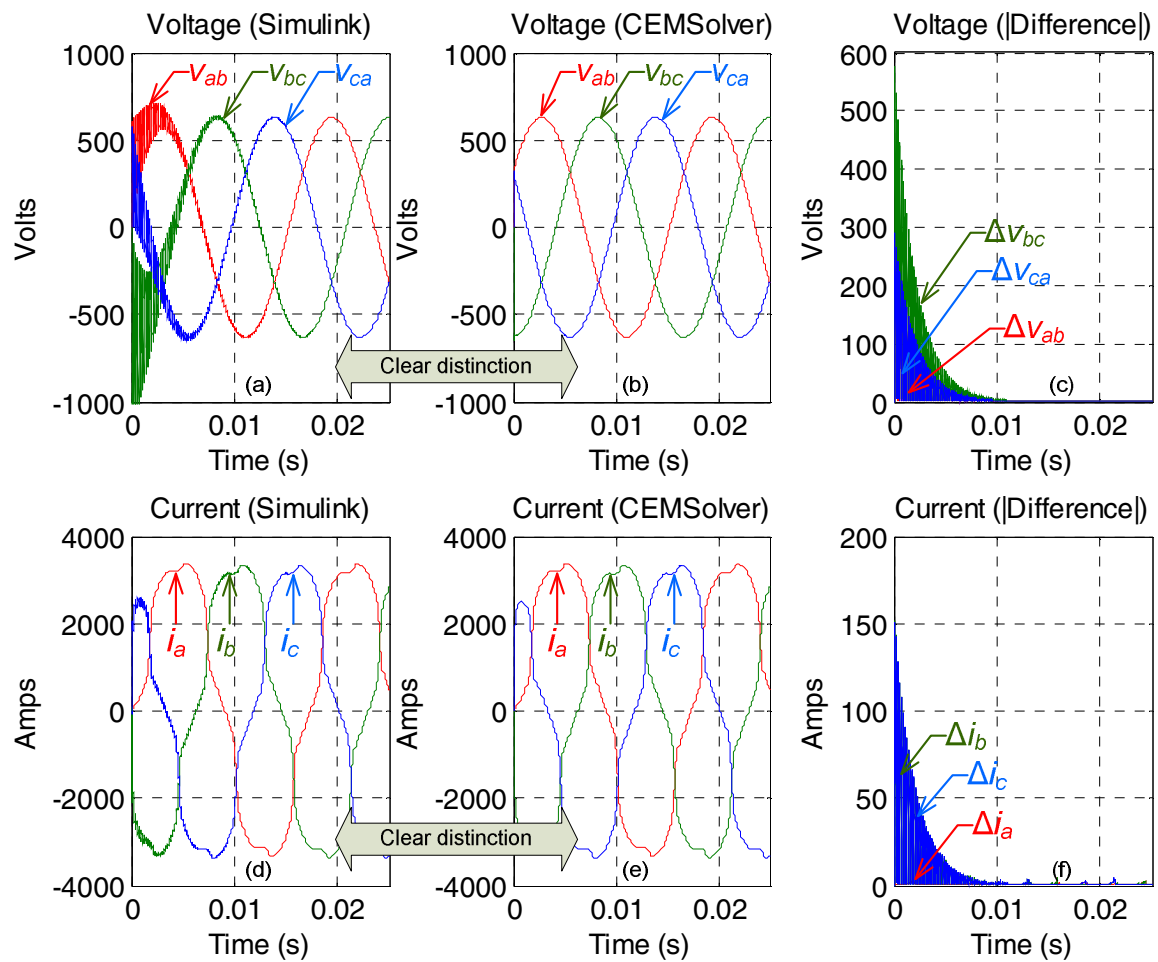

Figure 2 Voltage and current measured at GEN1 (A4) compared against Tustin integration.
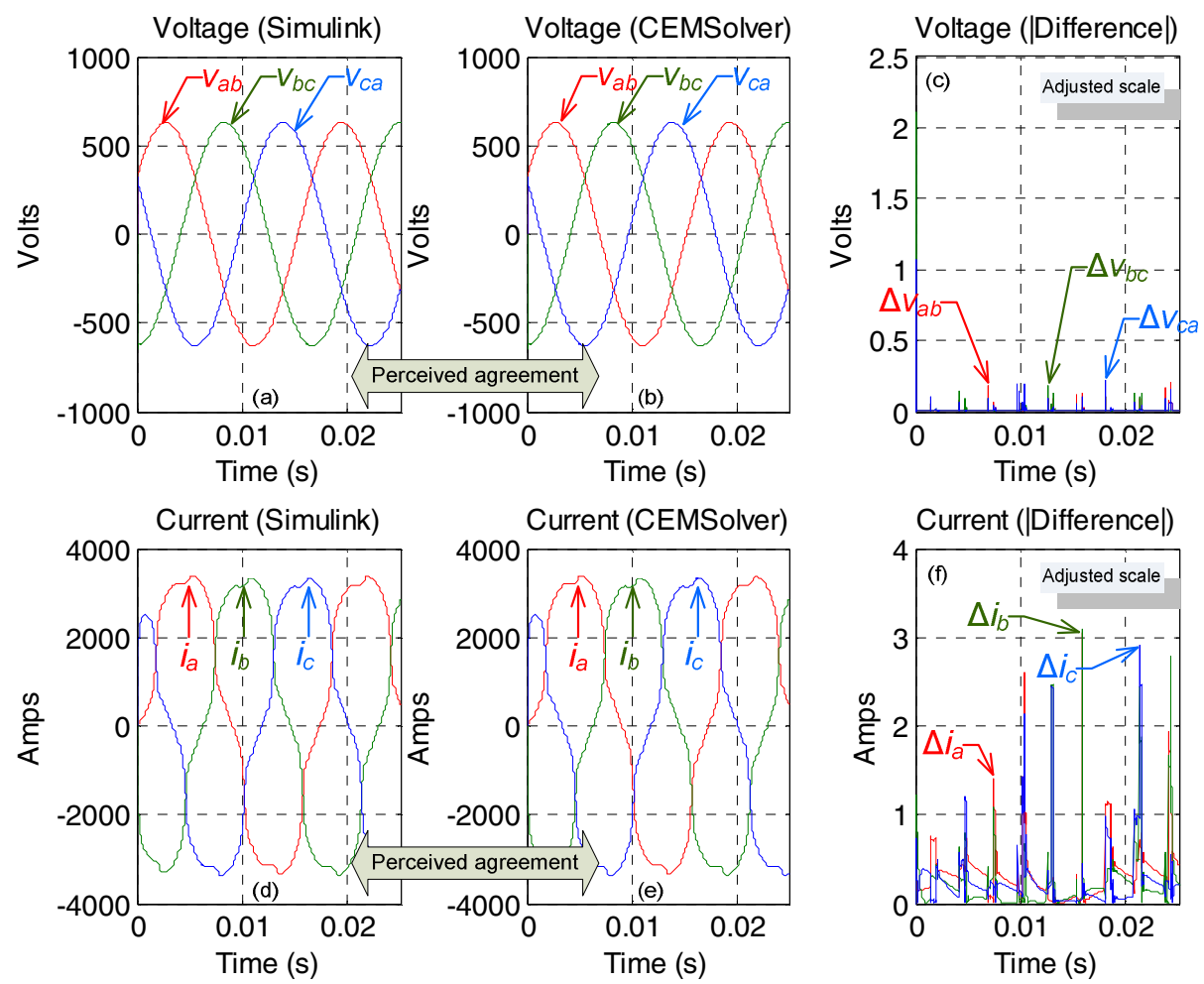

Figure 3 Voltage and current measured at GEN1 (A4) compared against Backward Euler integration. 

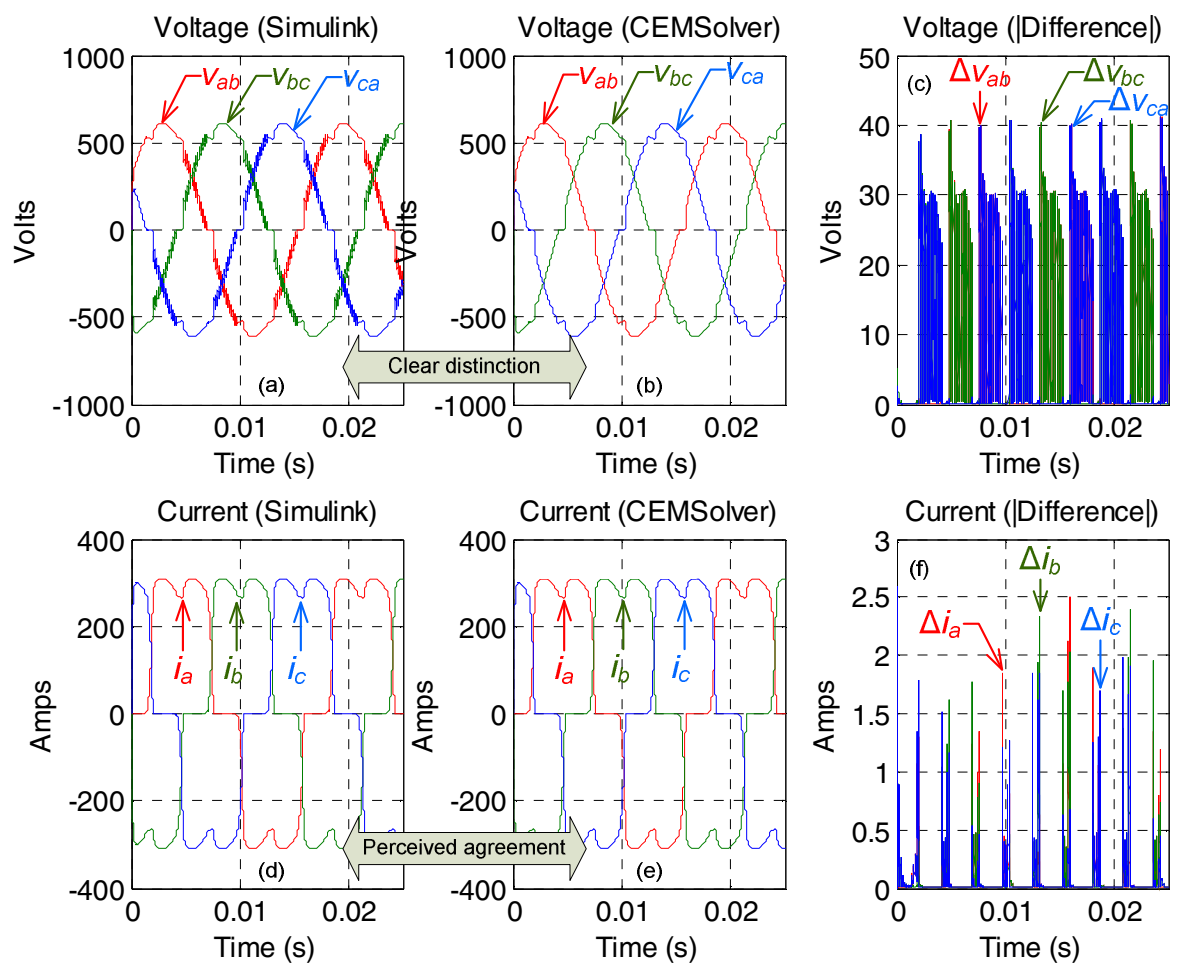

Figure 4 Voltage and current measured at M11 (E2) compared against Tustin integration.
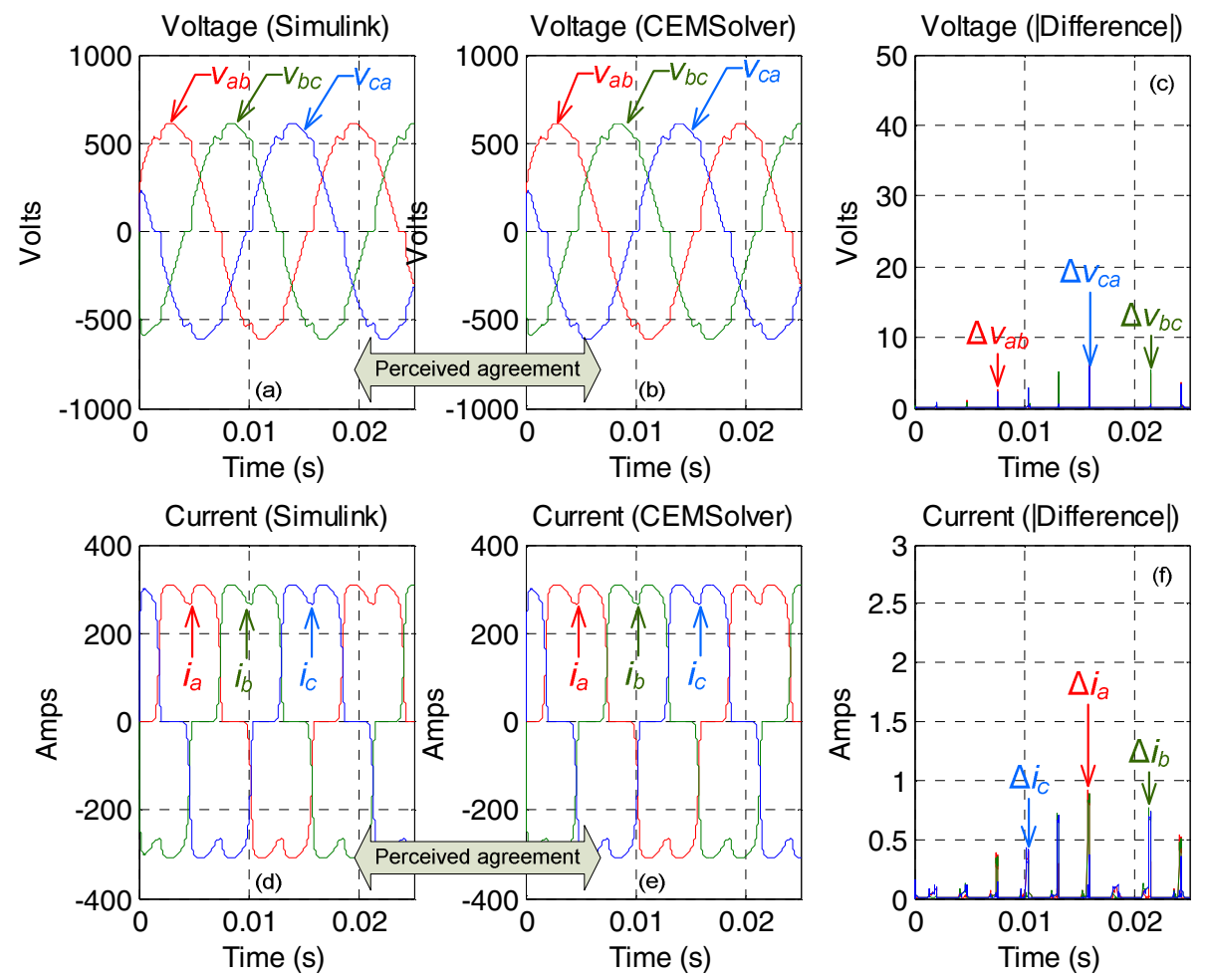

Figure 5 Voltage and current measured at M11 (E2) compared against Backward Euler integration. 

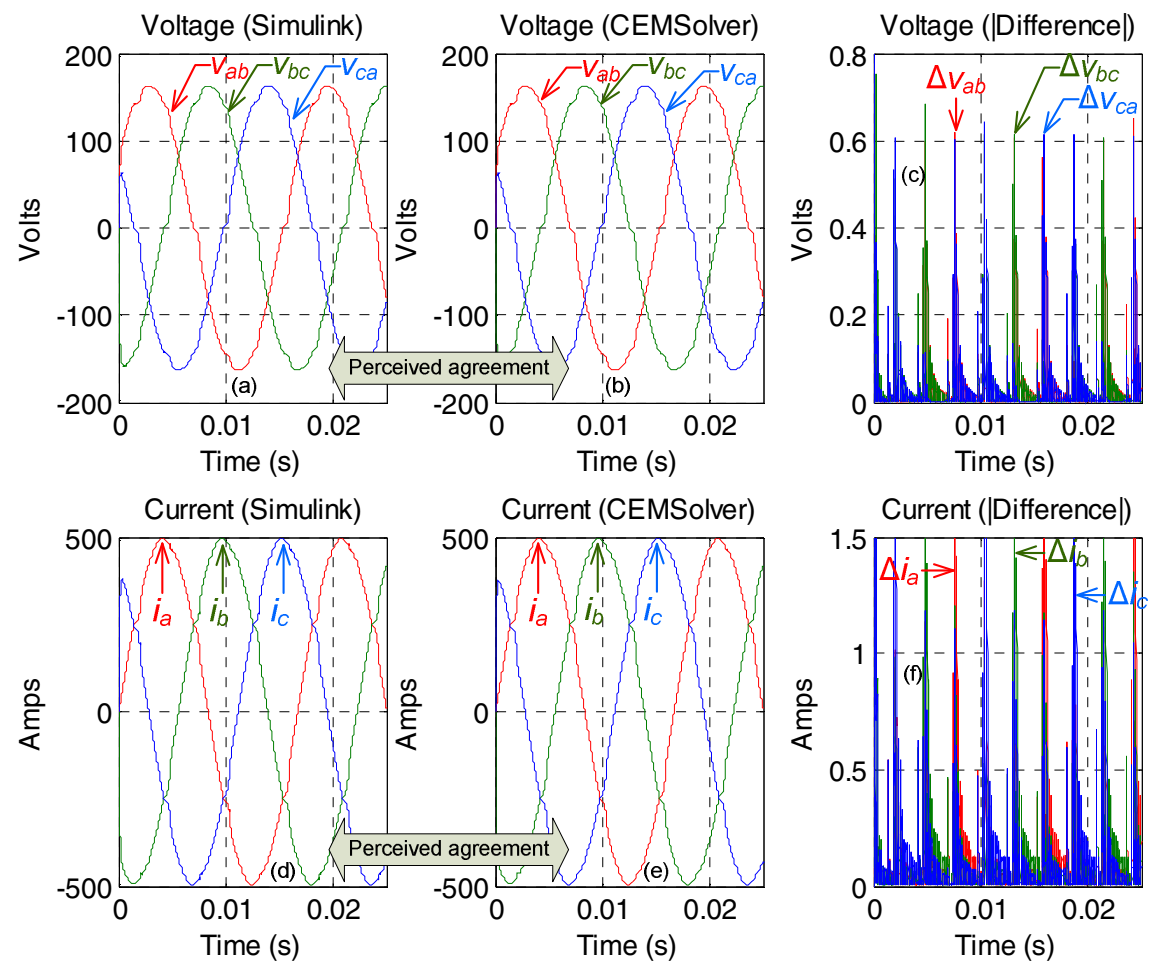

Figure 6 Voltage and current measured at T07 (F2) compared against Tustin integration.
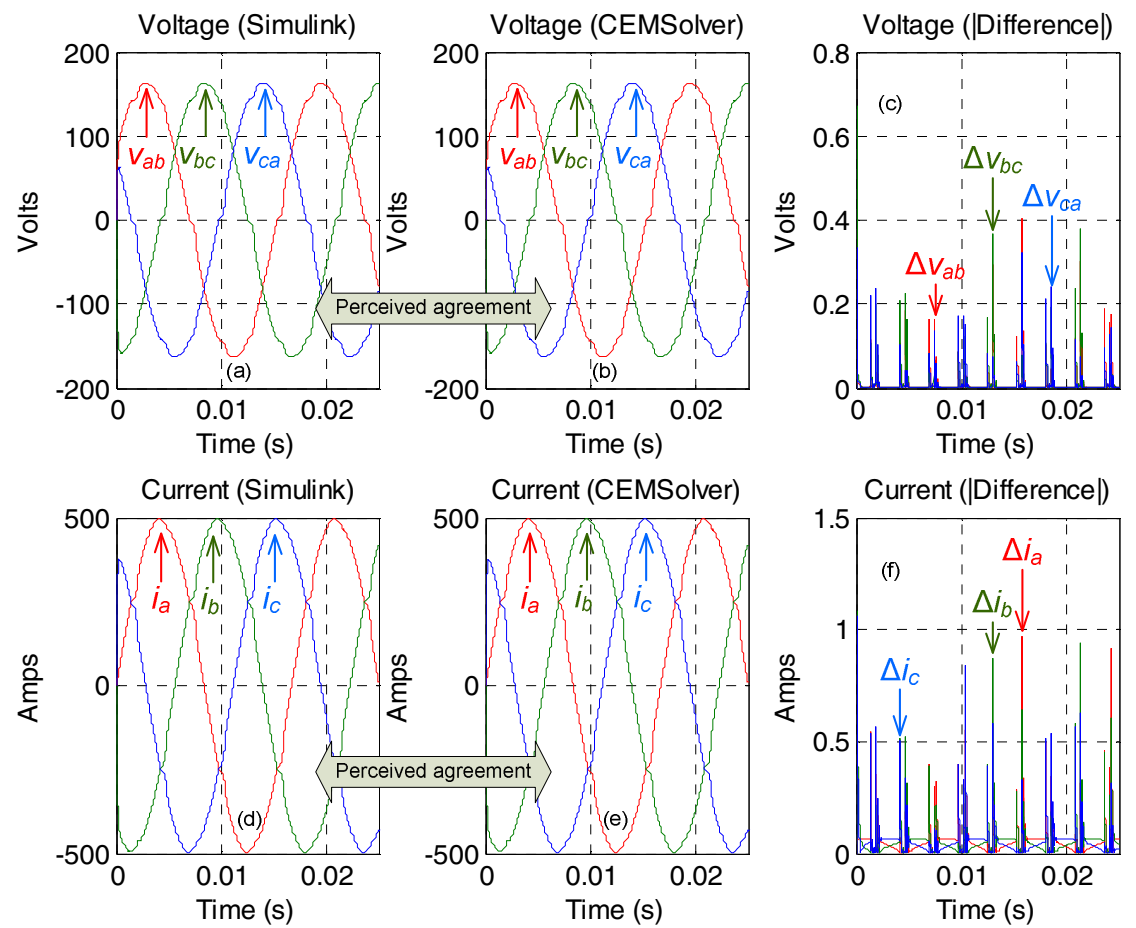

Figure 7 Voltage and current measured at T07 (F2) compared against Backward Euler integration 